\title{
Strates
}

STRATES Matériaux pour la recherche en sciences sociales

6 | 1992

La question de l'environnement : naissance d'un débat en Pologne

\section{La naissance de la démocratie dans la Pologne locale}

Bodhan Jalowiecki

\section{CpenEdition}

Journals

Édition électronique

URL : http://journals.openedition.org/strates/3013

DOI : $10.4000 /$ strates.3013

ISSN : $1777-5442$

Éditeur

Laboratoire Ladyss

Édition imprimée

Date de publication : 31 mars 1992

ISSN : 0768-8067

Référence électronique

Bodhan Jalowiecki, «La naissance de la démocratie dans la Pologne locale », Strates [En ligne], 6

1992, mis en ligne le 22 novembre 2007, consulté le 08 septembre 2020. URL : http://

journals.openedition.org/strates/3013; DOI : https://doi.org/10.4000/strates.3013

Ce document a été généré automatiquement le 8 septembre 2020

Tous droits réservés 


\title{
La naissance de la démocratie dans la Pologne locale ${ }^{1}$
}

\author{
Bodhan Jalowiecki
}

Dans la première moitié de l'année 1990 a été effectuée, dans le cadre du programme de recherche Développement régional-Développement local-Autonomie territoriale, une enquête sociologique par sondage ayant pour objet l'observation des changements sociaux qui sont intervenus dans les communautés locales. Les recherches portaient sur un échantillon de vingt et une localités situées dans différentes régions de la Pologne. Cet échantillon n'est certes pas représentatif, mais il est indéniablement suffisant pour pouvoir formuler des hypothèses documentées, d'autant que la majeure partie des communautés concernées avaient déjà été interrogées précédemment dans le cadre de ce même programme.

2 L'objet essentiel des investigations était de caractériser la scène politique locale et le processus de changement des élites après les élections du mois de mai 1990, qui avaient pour but de désigner les représentants des collectivités locales autonomes. Dans ce texte sont présentés, d'une manière très synthétique, les phénomènes et processus les plus caractéristiques observés au cours des enquêtes.

La formation des élites

Des conclusions générales, susceptibles d'être formulées à partir de l'observation des scènes politiques dans les localités faisant l'objet de l'étude, concernent avant tout quelques questions de nature politique. Pendant la période allant des élections parlementaires de juin 1989 (Diète et Sénat) aux élections administratives de mai 1990, on a pu observer - dans les communautés locales - le processus de formation de nouvelles élites locales. Les nouvelles élites qui ont pris le pouvoir dans les collectivités locales après leur victoire électorale sont encore très peu structurées. Elles tirent leur origine du mouvement Solidarité des années 1980/1981 et des structures clandestines mises en place dans les années quatre-vingt. La plupart des "Comités civiques" apparus au moment des élections ont donc une origine syndicale, bien que leur composition ait été beaucoup élargie par l'accession des représentants des milieux ruraux de "Solidarité des agriculteurs individuels ", ainsi que de personnes étrangères 
au mouvement Solidarité, mais qui ne s'étaient cependant pas salies par la collaboration avec l'ancienne nomenklatura.

4 À l'échelle locale, les membres des Comités civiques se recrutent principalement dans l'intelligentsia de formation humaniste plutôt que technicienne, ainsi que parmi les paysans (surtout à la campagne); la participation des ouvriers y est nettement plus faible.

5 Les Comités civiques n'ont qu'une faible structuration interne et n'ont pas de couleur politique déterminée. Les facteurs de cohésion sont avant tout - au moins verbalement - l'éthique de Solidarité, l'aspiration à de profonds changements dans la vie sociale et la lutte contre l'ordre ancien représenté auparavant par le Parti ouvrier unifié polonais (POUP), maintenant par l'ancienne nomenklatura. L'absence d'un adversaire bien défini fait que les attaques contre cette nomenklatura ont souvent un caractère chaotique, et que leur intensité dépend des configurations locales. Finalement, d'après les observations faites jusqu'à présent, cette lutte est plutôt un mot d'ordre qu'une activité dirigée contre des personnes concrètes, quoique l'on puisse également remarquer des cas de règlement de comptes envers les membres des anciennes élites.

6 Les Comités civiques n'ont pas de base sociale concrète. Les gens qui les soutiennent sont partout et nulle part. Les Comités civiques ne représentent pas non plus les intérêts déterminés d'une classe ou d'une couche sociale précise. Ils représentent les intérêts de la société tout entière, comme l'indépendance de la Pologne, qui n'ont que peu de rapports directs avec les intérêts locaux. Les Comités civiques sont parfois dominés par des groupes de personnes qui ne voient que leur intérêt particulier dans l'immédiat, après quoi ces groupes se scindent en sous-groupes antagonistes rivalisant entre eux, la plupart du temps sans merci.

7 L'amorphisme de la structure sociale fait que les intérêts plus durables, qui existent potentiellement, ne peuvent pas être clairement explicités. On peut constater empiriquement que certains Comités civiques représentent plutôt les intérêts des travailleurs salariés, d'autres ceux des paysans - mais plutôt des petits exploitants que des gros fermiers capitalistes. Les organes administratifs autonomes, qui sont une émanation des Comités civiques, ne peuvent aucunement bénéficier actuellement de l'appui de la couche des entrepreneurs capitalistes qui est en train de se former.

8 La formation d'une nouvelle structure sociale en Pologne, à la suite de la mise en place d'une économie de marché, sera un processus de longue durée. Pour l'instant, l'idéologie dominante des Comités civiques revêt encore un caractère socialiste On y prêche, en effet, des mots d'ordre préconisant l'égalitarisme, le bien des travailleurs, la lutte contre l'enrichissement au détriment d'autrui (comme s'il était possible de s'enrichir au détriment de soi-même), la qualité de la vie, les valeurs écologiques, des logements pour tous, un accès égal à l'instruction et à l'assistance médicale, etc., valeurs que le socialisme réel n'a jamais pu réaliser, mais qui imprègnent fortement la conscience sociale. Dans le même temps, le mot "socialisme » est condamné et chacun prend garde à ne pas le prononcer par mégarde. La rhétorique des Comités civiques porte donc l'empreinte du syndrome de Monsieur Jourdain, qui ne savait pas qu'il parlait en prose.

9 À la lumière des études réalisées, la discussion amorcée au sein des élites nationales de Solidarité sur l'avenir des Comités civiques n'est pas dépourvue de bon sens. Ne sont pas non plus dépourvues de fondement les craintes que les Comités civiques ne 
rappellent bientôt les anciens comités du POUP. Actuellement, ils ont déjà pris en charge un grand nombre de leurs fonctions: influence sur la politique de l'emploi, contrôle de l'activité des autorités locales, entre autres. Dans de nombreux cas, la nomination à un poste dépend de l'assentiment des Comités civiques. C'est là indéniablement un retour au système de la nomenklatura. L'acquisition d'une position quasi-monopolistique est déjà un fait dans de nombreuses agglomérations.

Il existe des risques de dégénérescence des Comités civiques comme de toute structure disposant d'une situation de monopole. Comme remède à cette situation, on a donc proposé le pluralisme politique au sein des Comités, avec la participation des représentants de diverses opinions ou tendances politiques. Ce serait peut-être une solution, mais qui n'est cependant pas du tout réaliste (sauf dans les grands centres urbains), car il n'existe pas - dans la majorité des communautés locales - de groupements politiques marquants et rien n'indique qu'il y en aura dans l'avenir le plus proche, faute de base sociale, c'est-à-dire de couches et classes sociales instruites susceptibles d'exprimer des intérêts contradictoires. Les différences entre les groupements respectifs ne sont que secondaires: elles renvoient à des débats idéologiques, dont l'origine remonte aux temps lointains d'avant la seconde guerre mondiale ou aux plus récents ressentiments à l'égard de groupements politiques tels que la Social-démocratie de la République polonaise (SDRP), c'est-à-dire les anciens communistes, le Parti démocrate (PD) et le Parti populaire polonais (PPP) - qui, d'après l'opinion publique, ne sont que d'anciens alliés des communistes, dissimulés sous de nouvelles couleurs. Les résultats des dernières élections administratives l'ont clairement montré. Il n'y a pas, en principe, d'intérêts nettement structurés sur la base de couches et classes sociales à statuts économiques variés : la société, dans sa plus grande partie, est représentée par la catégorie des travailleurs salariés, dont les différentes composantes luttent, il est vrai, pour une plus grande participation à la répartition du revenu national - quoique dans des limites très étroites. On est toujours en présence du conflit fondamental entre l'État employeur et les citoyens salariés. Un deuxième conflit objectif existe entre la ville et la campagne, c'est-à-dire entre les catégories urbaines (et rurales) de travailleurs salariés et les agriculteurs individuels. Ce conflit, qui revêt un caractère de plus en plus spectaculaire, ne peut trouver de solution dans un proche avenir, car il n'est possible de le limiter qu'en restructurant l'agriculture et en réduisant considérablement l'emploi dans ce secteur.

11 Par suite de l'absence d'intérêts réellement contradictoires sur le plan économique, les conflits politiques commencent à présenter un caractère de plus en plus personnalisé. Peu importe quelle fraction du mouvement Solidarité triomphera, car chacune d'entre elles se trouvera placée devant des problèmes similaires et devra les résoudre d'une manière identique, pour la bonne raison qu'il ne peut y avoir de programme alternatif dans la situation existante - tout au plus pourra-t-on mettre l'accent sur tel ou tel point.

12 Il y a un accord général sur la nécessité d'une restructuration économique, relative à la propriété, l'importance relative des différents secteurs de l'économie et la régionalisation. Tout le monde s'accorde à penser que, tout comme la concurrence est indispensable dans le domaine de l'économie, le pluralisme l'est en politique, et que la démocratie consiste dans le libre choix des représentants au Parlement et aux collectivités locales autonomes, dans le règne du droit, et dans l'existence d'au moins deux partis - un parti au pouvoir et un parti d'opposition. La majeure partie des 
représentants des élites politiques estiment qu'il faut un minimum d'interventionnisme de l'État pour empêcher la création de poches de misère. Le consensus porte sur ces objectifs formulés en termes généraux. Peu de gens sont cependant disposés à accepter les effets de la réalisation de ces postulats : faillite de branches entières de l'industrie, ruine d'un million ou plus d'exploitations paysannes, effondrement économique de régions et de nombre d'agglomérations, chômage massif et accroissement considérable des disparités sociales. Le capitalisme apparait au Polonais moyen sous l'aspect de magasins pleins de marchandises, dans lesquels chacun peut acheter presque tout; le capitalisme, c'est le niveau de vie de la République fédérale d'Allemagne ou de la Suède, mais non celui de la Turquie ou du Mexique où des millions de gens vivent dans l'indigence, où des milliers meurent de faim. Or la voie de la Pologne vers le capitalisme suivra plutôt le tracé propre au modèle caractéristique des pays du Tiers Monde. Entrer dans cette voie est une chance, mais seulement, malheureusement, pour les générations futures.

Le développement, à l'échelon national, de partis politiques représentant des intérêts, sinon contradictoires, du moins différents, est peut probable dans l'avenir le plus proche. Les groupements respectifs, comme nous l'avons déjà dit, sont divisés en général par une idéologie passéiste et par des querelles personnelles qui ne concernent personne en dehors des intéressés. Sur les scènes politiques locales, les partis présents à Varsovie et à la télévision n'existent pas et n'ont aucune audience. La seule chance non tant du pluralisme que du dualisme politique - est le partage de l'héritage de Solidarité entre deux formations qui n'auraient pas exactement les mêmes priorités dans la réalisation des réformes indispensables. Ce type de disposition de la scène politique peut cependant aussi bien stabiliser la situation, en canalisant le mécontentement social par l'acte de voter, que la déstabiliser par de fréquents changements de l'équipe au pouvoir.

Les Comités civiques se sont avérés capables de mener une campagne électorale efficace contre le régime déclinant. Réussiront-ils aussi facilement à gagner les élections sous l'étendard de la voie capitaliste de développement de l'économie, qui implique de nouvelles restrictions et l'abaissement du niveau de vie de groupes importants de travailleurs salariés? Seront-ils en mesure d'agir contre leurs propres intérêts immédiats? Peuvent-ils être un soutien pour un gouvernement qui réalise des réformes indispensables, bien qu'elles soient de moins en moins populaires ? Ou serontils, au contraire, un ferment antigouvernemental susceptible d'introduire efficacement des éléments anarchisants dans les rapports sociaux en Pologne? Le drame de la situation consiste dans le fait que la marge de liberté est très étroite. Si les tendances populistes prennent le dessus, il faudra repartir de zéro après quelques mois de détente apparente, mais l'économie sera alors au plus bas et le niveau de vie fléchira d'une manière tout à fait catastrophique.

La discussion sur l'avenir des Comités civiques ne peut pas être ramenée seulement à une controverse pour déterminer qui, grâce à eux, prendra le pouvoir dans un avenir très proche. La discussion sur les Comités civiques est dans une grande mesure une discussion sur l'existence de l'État.

Les analyses portant sur les scènes politiques locales ont également montré l'aspiration des Comités civiques à s'approprier la fonction de "force dirigeante», qui impose sa volonté aux autorités locales élues constitutionnellement, contrôle leurs actions, et réglemente les comportements des citoyens en bloquant la création d'initiatives en 
dehors du cercle des militants des Comités. Le système de cooptation et la nonacceptation de tous ceux qui donnent leur accord aux règles démocratiques du jeu politique, qui respectent les manières d'agir socialement admissibles dans la vie publique et privée, font que les Comités civiques se transforment en coteries et même en maffias. De nombreux responsables des Comités civiques considèrent ces derniers comme des tremplins qui leur permettront, de même qu'aux membres de leur famille, d'occuper des postes réservés jusqu'alors aux membres du POUP, et ils perçoivent le changement de régime non pas comme un changement des façons d'agir dans la vie publique, mais comme une simple relève des élites.

17 Le risque de dégénérescence des Comités civiques est perçu par les deux parties prenant part - sur la scène centrale - à la discussion portant sur leur avenir. Il en est qui, voyant les dangereuses dérives de ces Comités, optent pour leur dissolution.

Les principes de fonctionnement du système totalitaire sont enracinés profondément dans la conscience sociale, ce qui fait que les modèles de comportement dans la vie publique sont bien figés. Pour les changer, il faut beaucoup de temps et de persévérance pour appliquer un nouveau modèle de culture politique. Pour l'instant, on n'a rejeté que l'enveloppe extérieure de l'ancien système, alors que l'assise psychologique en est restée intacte, ce qui se manifeste dans la " tentation totalitaire » ressentie par de nombreux représentants des nouvelles élites dirigeantes, et pas seulement locales.

La lutte électorale

19 L'analyse de la campagne électorale dans les localités que nous avons étudiées montre qu'elle a été menée principalement par les Comités civiques, avec une certaine ampleur. Diverses techniques de propagande électorale ont été utilisées : réunions, meetings, kermesses, banquets. On a collé des affiches, distribué des prospectus, publié des annonces dans la presse, édité des numéros spéciaux de journaux, etc. Pour recommander les candidats, on s'est servi aussi bien des thèmes du programme que de leurs traits personnels, sans pour autant éviter de discréditer leurs adversaires en leur reprochant divers faits indignes.

20 Les Comités civiques ont engagé à ces fins de propagande d'importants moyens humains et financiers. Il semble qu'ils aient fortement surestimé la force de l'adversaire et sa capacité de mobilisation d'électeurs potentiels. Le principal facteur de la victoire électorale est le label Solidarité, qui jouit d'une connotation franchement positive dans la plus grande partie de la société. Voter pour les Comités civiques voulait dire se prononcer pour le changement, pour le départ des équipes usées et souvent même compromises, pour une chance d'améliorer les relations humaines et le climat moral, pour l'espoir d'un avenir meilleur pour le pays et les collectivités locales concrètes. En votant aux élections administratives de 1990, on ne votait pas tant pour un programme déterminé, car il n'y en avait pas du tout, mais plutôt pour le mot d'ordre Solidarité et les valeurs qui l'accompagnent. Un autre élément important expliquant le succès des Comités est la campagne électorale qui a été menée, comme nous l'avons dit, avec adresse et ampleur. Les Comités ont su parvenir jusqu'à la communauté locale et lui présenter leur candidat d'une manière attractive. Les rivaux des Comités civiques avaient cependant évalué la situation d'une manière erronée et n'ont pas fait beaucoup d'efforts, étant convaincus que leurs résultats ne seraient pas brillants, même s'ils se donnaient de la peine. Il s'est avéré, toutefois, que ceux des adversaires des Comités civiques qui ont mené une campagne électorale active ont souvent remporté la victoire. 
21 La campagne électorale a donc été menée sans grandes différences et n'a intéressé qu'un nombre restreint de supporters. Comme tout était, à vrai dire, connu d'avance, une grande partie de l'électorat, tant partisans qu'adversaires des Comités civiques, n'a tout simplement pas pris part au vote et la participation ne s'est élevée qu'à $42 \%$.

Les groupements liés à l'ancien système étaient convaincus a priori de ne pas pouvoir gagner la partie, aussi ont-ils assez souvent usé d'un camouflage consistant à ne pas se donner d'étiquette sur les listes électorales. C'était presque la règle dans le cas des candidats de la SDRP et du PPP, beaucoup plus rare lorsqu'il s'agissait du PD qui pour cette raison, entre autres, a essuyé la plus grande défaite. Cette tactique du camouflage avait été utilisée une seule fois par le Comité civique d'une localité, lors des élections parlementaires, et les résultats obtenus par Solidarité y ont été très médiocres.

La cause du faible intérêt éveillé par la campagne électorale et de la forte abstention était la trop grande généralité des programmes présentés par toutes les formations en présence, y compris les Comités civiques. C'est en cela que réside probablement la faiblesse majeure, tant des Comités civiques que des futures collectivités locales autonomes. Les élections administratives, c'est quelque chose de concret, et le conseiller municipal, dans les communautés locales de petite taille, ce n'est pas un député ou sénateur de Varsovie, lointain et peu connu, mais c'est le voisin, le camarade de travail, un des "nôtres ». Or les candidats utilisaient de grands mots, tels que démocratie, liberté, ou des plus petits, tels qu'esprit d'entreprise, autogestion. Ils se servaient souvent du langage des médias et des intellectuels de la capitale. Mais il y avait, à côté, la réalité : manque d'école, absence de magasin, rue défoncée, nombreux problèmes nécessitant une solution. Les candidats ne disaient pas qu'ils s'efforceraient de faire construire une station d'épuration des eaux pour que la laiterie voisine cesse de polluer la rivière qui baigne la ville, mais parlaient en revanche de protection de l'environnement. Ils ne disaient pas qu'ils rachèteraient des terrains à bâtir, les lotiraient et y mettraient en place des infrastructures, mais ils parlaient de construction d'habitations en général. Leur langage n'était guère adéquat à la réalité de la vie quotidienne, c'était le langage de ceux pour qui on avait voté maintes fois auparavant. C'était le langage des candidats du Front d'unité nationale ${ }^{2}$.

Si les candidats des Comités civiques s'adressaient de la sorte à leurs électeurs potentiels, ce n'était pas tout à fait de leur faute. Ils ne savaient simplement pas ce que pouvait être vraiment l'autonomie locale. Ils ne connaissaient pas les principaux actes juridiques devant réglementer leurs activités publiques, ni leurs compétences, car la loi délimitant les attributions de l'administration locale autonome et de l'administration gouvernementale n'a été votée que quelques jours à peine avant les élections. Dans deux des localités faisant l'objet de nos investigations, seuls les candidats à la fonction de conseiller municipal avaient suivi des cours sur l'autonomie locale, mais cet enseignement ne donnait pas non plus une information exhaustive sur cette dernière. Même lorsqu'ils avaient eu la possibilité de suivre un tel cours, beaucoup n'y étaient pas allés par manque de temps ou du fait des coûts du déplacement.

L'un des thèmes de la campagne électorale était la question des règlements de comptes avec le socialisme réel, avec la nomenklatura, avec les coteries précédentes. Cela n'a pas été spécialement développé dans les communautés locales étudiées, et si ce problème s'est parfois présenté pendant la campagne électorale des Comités civiques, ce n'était, en de nombreux cas, qu'un écho des problèmes traités par les médias. Certains se sont servis de ces mots d'ordre, car il leur semblait qu'il fallait le faire, mais 
ils s'y prenaient avec prudence et parfois aussi avec une certaine gêne. Dans les communautés locales, une personne de la nomenklatura est très souvent un camarade d'école, un copain de travail, un voisin, qui savait vivre et aidait les autres à vivre. On a affaire à Jasio ou Franio, non à un représentant impersonnel de la terrible nomenklatura. La consigne donnée de lutter contre l'ordre ancien avait également été fortement affaiblie par le fait que l'organisation politique qui le représentait, le POUP, avait quasiment disparu de la scène publique. On pouvait donc lutter contre une ombre mais pas contre un adversaire réel, ce qui diminuait grandement la dynamique de ce conflit.

Le drame de la société issue du socialisme réel, ou si l'on préfère de la société postcommuniste, consiste non seulement dans le fait que plusieurs millions de personnes se trouvaient dans la nomenklatura des organisations du Parti à des échelons variés, mais aussi dans le fait que la société tout entière a été éduquée dans un système qui contraignait les gens à se déplacer dans la réalité quotidienne par des chemins tortueux, à faire des combines et des louvoiements, à éluder et à enfreindre la loi, dans un système qui a déshabitué les gens à avoir du respect pour le travail, la concurrence, l'enrichissement, l'État et les institutions nationales. Rares étaient ceux qui avaient su résister aux influences, néfastes au tissu social, du socialisme réel. Seule une profonde rééducation de toute la société est en mesure de modifier cette situation. Qui a gagné les élections?

Bien que, d'une façon générale, les résultats du scrutin aient démontré une nette supériorité des Comités civiques dans la mouvance de Solidarité, ils sont cependant diversifiés. Dans quatorze cas étudiés (sur vingt et un), les Comités civiques ont obtenu la majorité absolue des suffrages, ce qui revient à dire qu'ils détiennent entièrement le pouvoir. Cependant, dans deux localités, cette supériorité n'est pas si nette: il suffit que deux conseillers municipaux ne viennent pas à la session pour que l'opposition devienne majoritaire. Dans une localité, le Comité civique n'a obtenu que le tiers des suffrages, dans quatre autres agglomérations environ deux cinquièmes des voix et dans une autre exactement la moitié. N'ayant pas la majorité absolue dans cinq localités, les Comités civiques sont obligés de faire des alliances avec d'autres groupements politiques. Cela apparaît à l'occasion de l'élection des membres des présidiums des conseils, des bourgmestres et des maires de communes rurales.

Il est cependant possible de donner des exemples où les Comités civiques Solidarité ont obtenu la majorité des voix, mais où, aux postes de bourgmestres et de maires de communes rurales, ont été élus des spécialistes connus pour leur appartenance aux anciennes élites dirigeantes. Les nouvelles collectivités locales autonomes sont donc disposées à consentir à des compromis, à assurer une certaine continuité et à donner la préférence à l'expérience et au savoir-faire, qui sont parfois l'apanage des représentants de l'ancienne nomenklatura. Ce type de comportement est plus fréquent dans les petites communautés, car on y:manque de gens ayant les qualifications voulues pour pouvoir assumer une fonction dans l'administration autonome.

On peut donc dire qu'à la suite des élections administratives a eu lieu un changement considérable des élites et que des gens tout à fait nouveaux ont accédé au pouvoir. Mais d'autre part, une partie des anciennes élites dirigeantes complète - dans une relative harmonie - les élites nouvellement venues. Ces élites doivent donc coexister dans nombre de localités. Plus souvent encore, les positions des anciennes élites d'influence sont restées intactes : bien enracinées dans les milieux locaux, elles continueront à 
avoir une influence réelle sur l'évolution des processus sociaux dans la Pologne locale, grâce à des collusions amicales et familiales.

Parmi les nouveaux conseillers municipaux qui se proclamaient indépendants aux élections administratives, on trouve ainsi des représentants des anciennes élites dirigeantes et surtout de ces élites d'influence, c'est-à-dire des personnes qui n'assumaient aucune fonction formelle dans les organes du pouvoir local, mais jouissaient d'une grande popularité et d'un grand prestige en raison du rôle social qu'elles remplissaient.

On ne peut pas parler de révolution dans la Pologne locale, du moins dans les communautés qui ont fait l'objet de nos enquêtes, mais il y a eu néanmoins une nette évolution: les élections administratives ont provoqué, en effet, l'apparition d'une multitude de gens nouveaux, dont une partie au moins jouera un rôle important dans le processus des changements sociaux.

Les partis politiques n'ont exercé qu'un rôle minime lors de ces élections. Le seul parti qui a marqué sa présence d'une manière apparente, dans certaines localités, est le PPP. Le nombre de mandats acquis par ce parti est cependant moins important que ne le laissaient pressentir sa structure organisationnelle et sa logistique, héritées intactes de l'époque de l'ordre ancien. Le PPP est considéré comme un parti dissimulé sous de nouvelles couleurs, et de plus il a été affaibli par des querelles intestines menées à l'échelon central juste avant les élections. Le PD n'existe presque pas sur la scène politique locale. Il en est de même de la SDRP : elle n'a pas émergé du tout dans la plupart des localités faisant l'objet des études durant les élections. Les nouveaux partis de droite, tels que l'Union nationale chrétienne ou la Confédération de la Pologne indépendante, sont totalement absents dans les localités en question, à l'exception de Tychy et Pulawy. C'est seulement à Pulawy et dans les quartiers de Varsovie que nous avons étudiés que ces deux dernières formations politiques ont obtenu chacune un mandat.

Les Comités civiques avaient un adversaire réel aux élections administratives: les candidats indépendants qui étaient représentés au moins en aussi grand nombre que ceux de Solidarité. Dans de nombreuses localités, ils étaient considérés comme les concurrents les plus menaçants. En général, comme on l'a déjà dit, les candidats indépendants provenaient des anciennes élites d'influence, c'est-à-dire qu'ils n'appartenaient pas forcément aux précédentes structures dirigeantes. Si les anciens membres du POUP étaient nombreux parmi eux, il s'agissait habituellement de simples membres, qui auparavant n'avaient pas accès aux milieux dirigeants et sont maintenant rejetés par les nouvelles élites. Comme leur appartenance à l'ex-POUP les éliminait automatiquement du mouvement Solidarité, ils posaient individuellement leur candidature aux élections, désireux de continuer à agir en faveur de la communauté locale, ou tout simplement de se confirmer eux-mêmes dans l'action. Dans nombre de cas, ils ont réussi : ils sont passés au tamis des élections. Les milieux dans lesquels ils vivaient les ont acceptés.

De nombreux facteurs, se recouvrant les uns les autres, ont contribué à la victoire des Comités civiques. Un rôle important, quoique très peu décisif dans la Pologne locale, a été joué par le label Solidarité, associé au processus de changement intervenant dans notre pays et à la nouvelle réalité politique. L'influence en a été déterminante dans les grandes villes, où personne ne connaissait les candidats. Un autre facteur a été leur campagne électorale, généralement bien menée, avec des moyens modernes, qui a 
permis de bien présenter les candidats aux divers milieux. Il était important de parvenir personnellement jusqu'aux électeurs lorsque le candidat était peu connu ou qu'il n'habitait pas dans sa circonscription électorale. Dans une moindre mesure a joué le programme électoral des Comités civiques, qui presque partout ne comportait que des banalités peu éloquentes, souvent répétées, quasiment dans les mêmes termes, à l'occasion des élections pour désigner les membres des conseils populaires de la République populaire de Pologne. À la victoire des Comités civiques a contribué également la situation conflictuelle entre les communautés et l'administration locale précédente, surtout quand celle-ci était particulièrement incapable ou corrompue.

Le rôle de l'Église n'est pas négligeable non plus : bien qu'elle ne se soit pas engagée, en général, dans la lutte électorale, elle a été cependant plutôt favorable aux candidats des Comités civiques, mais surtout elle a encouragé à prendre part au vote, ce qui a contribué dans une certaine mesure à l'augmentation de la participation électorale.

Une situation relativement stable dans la localité concernée et l'absence de conflits visibles entre la communauté locale et le pouvoir précédent n'ont guère été favorables aux Comités civiques Solidarité. Le radicalisme à outrance de certains de ces Comités a également effrayé les électeurs qui ne voulaient pas intensifier les querelles personnelles, encourager des règlements de comptes et déstabiliser outre mesure l'équilibre local.

Il faut enfin faire état d'un facteur qui a pu agir en faveur des deux camps, mais qui était plutôt favorable aux anciennes élites. Les choix des votants dans la Pologne locale ont été fortement personnalisés : gagnait en général celui qui était solidement enraciné dans le milieu concerné, était bien connu de ses électeurs, avait déjà eu la chance de faire ses preuves sur le plan social. Beaucoup de mandats ont été obtenus, par exemple, par des candidats qui ont su convaincre les électeurs qu'ils savaient faire quelque chose de concret, concourir à la construction d'une école, d'une route, d'un réseau de distribution de gaz, etc. Dans ces cas-là, l'appartenance organisationnelle était d'une importance minime ou même nulle.

L'absence d'une forte majorité en faveur des Comités civiques de Solidarité ou une composition diversifiée du conseil municipal, sans qu'aucun des camps ne soit majoritaire, seront, semble-t-il, des facteurs positifs pour l'activité du conseil et favoriseront la présentation de diverses options. L'attention des conseillers municipaux sera, par la force des choses, dirigée plus sur les questions d'intérêt général que sur les querelles intestines. Dans ce cas-là, il y a une chance pour que les groupements politiques respectifs se consolident intérieurement face à un concurrent puissant. Chacun voudra, en effet, prouver à la communauté locale qu'il est le meilleur.

Une situation tout à fait différente peut se présenter dans les conseils municipaux dominés par les Comités civiques. Certains de leur position et de l'appui social, les représentants pourront se concentrer sur des luttes intestines, qui useront leurs forces et seront une entrave au règlement des problèmes importants. On a pu observer de tels faits dans deux localités. Dans l'une d'elles, il n'a pas été possible d'élire le bourgmestre pendant un mois entier : sept candidats menaient entre eux une lutte sans merci pour accéder à cette fonction. Dans la seconde, le bourgmestre a été élu, mais une partie du Comité civique du lieu refuse d'accepter ce choix et organise des manifestations, des piquets de grève.

Il vaut également la peine de se demander qui a vraiment remporté la victoire sur les scènes politiques de la Pologne locale, car l'affirmation selon laquelle les Comités 
civiques ont gagné, dans la plupart des cas, ne signifie pas grand-chose. En effet, quel est le visage politique des membres de ces Comités et des conseillers municipaux qu'ils ont mis en place? La plate-forme électorale commune des Comités civiques, c'est le refus de la réalité existante et le désir de contribuer à la création d'un nouvel ordre social. Mais de quel ordre s'agit-il ? Il n'est pas facile de le dire. Outre les principes fondamentaux se rapportant à la doctrine sociale de l'Église et à l'éthique de Solidarité, il était beaucoup question de problèmes concrets devant être réglés. C'est là évidemment le trait caractéristique des élections au niveau de l'administration locale autonome. Ces déclarations en disent cependant plus long sur les principes moraux et sur un certain pragmatisme que sur le visage politique et la forme concrète de ce nouvel ordre social. Les programmes électoraux des Comités civiques contiennent implicitement un fond socialisant. En fait, nous sommes en présence d'un programme d'ordre social socialiste sans "socialisme». Le camp adverse fait des déclarations identiques. Il semble donc que les Polonais sont bien plus divisés par le passé que par leur attitude envers l'avenir.

41 La participation électorale est le dernier problème qui a donné lieu à de fréquentes discussions. Dans de nombreuses communautés locales étudiées, elle a été supérieure à la moyenne nationale. Le nombre de candidats pour un mandat a été le facteur déterminant l'accroissement de la participation au scrutin. Un nombre plus important de candidats faisait baisser les abstentions. L'engagement personnel en faveur d'un candidat a exercé également une influence positive sur la participation au vote. Les causes des abstentions ont été, comme on l'a déjà évoqué, variées et très complexes.

Tout d'abord n'ont pas pris part au scrutin les personnes qui ne s'intéressent pas du tout aux affaires publiques. Le deuxième groupe se compose de partisans de Solidarité, qui étaient convaincus que la cause était gagnée et que ce n'était pas la peine d'aller voter. Une partie des électeurs n'ont pas voté faute de connaître les candidats, ne voulant pas se prononcer pour une formation politique quelconque à l'occasion des élections administratives. D'autres n'ont pas participé au scrutin car aucun des candidats ne leur convenait, et pour cause - ils les connaissaient très bien. Il ne valait pas non plus la peine d'aller voter lorsqu'il n'y avait qu'un seul candidat dans la circonscription. Une partie des citoyens étaient mécontents du fait qu'ils n'avaient pas leur mot à dire sur la composition du conseil municipal de l'ensemble de la commune, mais devaient seulement voter pour un candidat. La loi électorale elle-même a été dans un certain sens source d'abstentions. Une partie des électeurs n'ont pas voté parce que la politique du gouvernement ne leur convenait pas, les agriculteurs à cause de la prétendue sous-estimation de l'agriculture par l'État, l'ancienne nomenklatura du fait de la persécution organisée injustement - selon elle - à son égard. Il y a également une catégorie d'électeurs n'ayant pas participé au vote à cause du manque de stabilité dans notre pays et de l'existence de troubles sociaux (la grève des cheminots, en mai dernier). Face aux menaces pesant sur eux, les élections sont devenues une question tout à fait secondaire. Ces attitudes sont confirmées par des déclarations des personnes interrogées au cours de notre enquête. Comme les causes sont nombreuses, il ne faut donc pas tirer de conclusions nettement politiques du fait que la participation électorale a été faible. Un si grand nombre d'abstentions - environ la moitié de l'électorat de la Pologne locale - pose le problème de la légitimation sociale de l'autonomie territoriale. Les élections n'ont pas fourni cette légitimation. Dans certaines circonscriptions, les conseillers municipaux ont été élus en ne recueillant qu'une quinzaine de voix. Ils ne pourront obtenir cette légitimation qu'en œuvrant en 
faveur de leurs communautés. Il est à souhaiter que cela se produise le plus vite possible.

Les nouveaux conseils municipaux se composent principalement de personnes ayant une instruction supérieure, ce qui est une circonstance positive. On constate l'absence presque totale de femmes : il n'y en avait que très peu parmi les candidats, et elles n'ont pas eu, en règle générale, la faveur des électeurs. Les nouveaux conseils municipaux autonomes sont masculinisés à l'extrême, ce qui n'est pas un phénomène positif. Dans la République populaire de Pologne, on prenait soin, du moins formellement, de faire participer les femmes à la vie publique, ceci au moyen de quotas lors des élections. La démocratie élimine presque totalement, malheureusement, les femmes de la vie publique.

Le démarrage de la démocratie locale est difficile. Il n'est pas du tout facilité par la très mauvaise situation économique, les revendications croissantes des paysans et de diverses catégories de travailleurs salariés, la déstabilisation progressive des structures de l'État et la lutte pour le pouvoir entre différents groupements qui se réclament de Solidarité. Les nouveaux conseils municipaux autonomes commencent à agir en l'absence d'un système de financement des budgets locaux. Dans la plupart des localités, la caisse est vide mais les besoins sont immenses. À l'heure actuelle, dans de nombreuses communes il y a à peine assez d'argent pour couvrir les dépenses courantes les plus urgentes. Il n'est absolument pas question de réaliser les investissements pressants. Dans cette situation, il n'y a pas de possibilité de répondre aux attentes sociales en matière d'amélioration sensible des conditions d'existence. Le seul changement d'élites dirigeantes suffira-t-il et pour combien de temps?

\section{NOTES}

1. Version française revue par Martine Berger.

2. Organisme fantoche créé en 1950 par le parti communiste.

\section{RÉSUMÉS}

Ce texte présente les principaux processus observés lors des enquêtes réalisées en 1990 dans 21 localités polonaises afin de caractériser la nouvelle scène politique locale. Le démarrage de la démocratie locale s'avère difficile : avec les élections de mai 1990, de nouvelles élites dirigeantes sont apparues, mais les principes du fonctionnement du système totalitaire sont profondément enracinés dans la conscience sociale.

The emergence of democracy in local Poland 
In Poland, the new political scene is revealing some major trends which can be reviewed through the analysis of a 1990 survey led into 21 local communities. The fact is that the setting-up of local democracy has turned out to be rather difficult : new elites have emerged in may 1990, but the principles at work in the totalitatian system keep being deeply rooted in social consciousness.

\section{INDEX}

Mots-clés : Pologne, systèmes locaux de pouvoir, démocratie locale, élites dirigeantes, géographie politique, comportements électoraux, political geography

Keywords : Poland, local authority reforms, local management

\section{AUTEUR}

BODHAN JALOWIECKI

Professeur à l'université de Varsovie, il est directeur adjoint de l'Institut européen du développement régional et local de Varsovie. Il exerce des fonctions de conseiller auprès du ministre de l'Administration. Il édite en Pologne des textes en langue française, et a publié des articles dans des ouvrages édités en France (comme Banlieues d'aujourd'hui) (L'Harmattan) ou Espaces et travail clandestin (Solange Montagné-Villette (dir.), Masson). 